\title{
Modelling water molecules inside cyclic peptide nanotubes
}

\author{
Prangsai Tiangtrong $^{1,2} \cdot$ Ngamta Thamwattana $^{3} \cdot$ Duangkamon Baowan $^{1,2}$
}

Received: 12 February 2015/Accepted: 11 March 2015/Published online: 27 March 2015

(C) The Author(s) 2015. This article is published with open access at Springerlink.com

\begin{abstract}
Cyclic peptide nanotubes occur during the selfassembly process of cyclic peptides. Due to the ease of synthesis and ability to control the properties of outer surface and inner diameter by manipulating the functional side chains and the number of amino acids, cyclic peptide nanotubes have attracted much interest from many research areas. A potential application of peptide nanotubes is their use as artificial transmembrane channels for transporting ions, biomolecules and waters into cells. Here, we use the Lennard-Jones potential and a continuum approach to study the interaction of a water molecule in a cyclo[(-DAla-L-Ala $\left.)_{4}-\right]$ peptide nanotube. Assuming that each unit of a nanotube comprises an inner and an outer tube and that a water molecule is made up of a sphere of two hydrogen atoms uniformly distributed over its surface and a single oxygen atom at the centre, we determine analytically the interaction energy of the water molecule and the peptide nanotube. Using this energy, we find that, independent of the number of peptide units, the water molecule will be accepted inside the nanotube. Once inside the nanotube, we show that a water molecule prefers to be off-axis, closer to the surface of the inner nanotube. Furthermore, our study of two water molecules inside the peptide nanotube
\end{abstract}

Ngamta Thamwattana

ngamta@uow.edu.au

Duangkamon Baowan

duangkamon.bao@mahidol.ac.th

1 Department of Mathematics, Faculty of Science, Mahidol University, Bangkok, Thailand

2 Centre of Excellence in Mathematics, CHE, Si Ayutthaya Rd, 10400 Bangkok, Thailand

3 School of Mathematics and Applied Statistics, University of Wollongong, Wollongong, NSW 2522, Australia supports the finding that water molecules form an array of a $1-2-1-2$ file inside peptide nanotubes. The theoretical study presented here can facilitate thorough understanding of the behaviour of water molecules inside peptide nanotubes for applications, such as artificial transmembrane channels.

Keywords Cyclic peptide nanotubes - Water molecules · Artificial transmembrane channels - Interaction energy . Offset positions $\cdot$ Lennard-Jones potential

\section{Introduction}

The discovery of self-assembly peptides has had a great impact on many areas of study, such as biomedical nanotechnology (Zhang 2003; Scanlon and Aggeli 2008; Shoseyov and Levy 2008), cell culturing (Zhang 2003) and molecular electronics (Scanlon and Aggeli 2008; Shoseyov and Levy 2008). This is due to the spontaneous assembly process of peptides into stable nanostructures including dipeptide (Scanlon and Aggeli 2008), lego peptide (Zhao and Zhang 2007), surfactant peptide (Zhang 2003), carpet peptide (Zhang 2003) and cyclic peptide (Scanlon and Aggeli 2008). In this paper, we study a cyclic peptide nanotube arising from the self-assembly process of cyclic peptides stacking to form a tubular structure. The experiment of Ghadiri et al. (1993) shows that ring-shaped cyclic peptides can be formed by an alternating D- and Lalpha-amino acids, and the hydrogen bonding between the two amino groups dominates the interaction between these cyclic rings forming a stable peptide nanotube. The amino acid side chains of a cyclic peptide can be used to determine the characteristics of the outer surface of the tube, while the properties of the inner surface of the tube are 
determined by the peptide backbone (Scanlon and Aggeli 2008). Therefore, the properties of the outer surface of a cyclic peptide nanotube can be determined by the functional side chains and the inner diameter of the tube can be deduced based on the number of amino acids forming the cyclic peptide. These and other properties of cyclic peptide nanotubes, such as the ability to resist high and low temperatures, detergents, and denaturants (Dinca et al. 2008), have led to many proposed applications for peptide nanotubes, one of which is their use as transmembrane channels for transporting ions, biomolecules and water molecules into cells. While Rahmat et al. (2011) study the interactions of ions and ion-water clusters inside peptide nanotubes, this paper focuses on interactions involving water molecules and their arrangement inside the peptide nanotubes.

There are a number of studies in the literature that concern the interactions of water molecules and peptide nanotubes. In Kim et al. (1998), Hwang et al. (2009), Khalfa et al. (2009), Khurana et al. (2008), Delemotte et al. (2008), it has been shown that due to the hydrophilic or hydrophobic specialities of the outer surface, the cyclic peptide nanotube can exist stably in water. Tarek et al. (2003) state that the translocation of water molecules inside the cyclic peptide nanotube consisting of eight cyclo[(L-Trp-D-Leu $)_{3}-\mathrm{L}-\mathrm{Gln}$ D-Leu] units mediates the hydrogen bond between each cyclic peptide unit intermittently. However, this process does not affect the interactions between each peptide ring and does not change the average distance between each cyclic peptide unit. In addition, Tarek et al. (2003) show that the encapsulation rate of the water molecules depends on the radius of the nanotube and the behaviour of the water molecules inside a peptide nanotube depends on the environment and their structures inside the tube.

For the motion behaviour of water molecules in a cyclo[(-D-Phe-L-Ala $\left.)_{n=4^{-}}\right]$peptide nanotube, Zhu et al. (2008) use a COMPASS force field to show that due to the stacking arrangement of antiparallel rings, water molecules do not diffuse unilaterally, but do so in one dimension. Song et al. (2013) study the dynamics of $\mathrm{Na}^{+}$transportation in a cyclic peptide nanotube of $8 \times(\mathrm{WL})_{4} / \mathrm{POPE}$ that contains water molecules. Their results show that $\mathrm{Na}^{+}$and the bare carbonyls induce electrostatic potentials which affect the arrangement of water molecules forming " $D$ defects" located in the regions of gaps 1 and 7 of the cyclic peptide nanotube. Using the mean force profile for water molecules along the tube axis and the cylindrical distribution functions at different zones of a nanotube, Liu et al. (2010) suggest that steric constraints are the reason for the water molecules to form a water chain inside the nanotube. Liu et al. (2010) also investigate the structure of a water chain in the $8 \times$ cyclo- $(\mathrm{WL})_{4}$ peptide nanotube embedded in POPE lipid bilayer. Their results show the wavelike pattern of water chain along the tube axis arraying in the form of a $1-2-1-2$ file. Their results agree with Engels et al. (1995) who also state that the water molecules prefer to be in the free space between each peptide ring. However, results of Song et al. (2013), Liu et al. (2010), Engels et al. (1995) contradict the findings of Raghavender et al. $(2009,2010)$ that in tubes with diameters ranging from 5.2 to $7.7 \AA$, water molecules align in a single file along the long axis of a peptide nanotube.

To consider the interaction and the arrangement of water molecules inside a peptide nanotube, we propose a mathematical model based on the Lennard-Jones potential and a continuum approach. The continuum approach using the Lennard-Jones potential was first introduced by Girifalco (1991) for the interaction between two $\mathrm{C}_{60}$ molecules. Subsequently, the approach has been successfully adopted in other studies, such as Baowan and Hill (2007) and Cox et al. (2007a, b, 2008) to obtain explicit analytical criteria for the interaction potentials involving carbon nanostructures. Furthermore, studies of interactions involving biological materials, such as lipid bilayers, lipid nanotubes and liposomes (Baowan et al. 2012; Baowan and Thamwattana 2014; Baowan et al. 2013), have also indicated that this approach produces results in good agreement with intensive computational studies. For the study of peptide nanotubes, Rahmat et al. (2011) adopt the Lennard-Jones potential and the continuum approach to model the interactions of a peptide nanotube with an ion, an ion-water cluster and a fullerene $\mathrm{C}_{60}$. The results obtained are shown to be consistent with experiments and molecular dynamics simulations. With this in mind, this paper adopts this approach to investigate the potential use of peptide nanotubes as artificial channels for transporting water molecules. Through this approach, we assume that a peptide nanotube is made up of a finite number of peptide units and that each unit of the nanotube comprises an inner and an outer tube. We also assume that a water molecule is made up of a sphere of two hydrogen atoms uniformly distributed over its surface and a single oxygen atom at the centre. Further, we adopt the concepts proposed by Cox et al. (2007a, b) to explain the encapsulation behaviours of a single atom, a spherical molecule, and a water molecule in a peptide nanotube. We also determine the preferred offset locations of these structures inside the cyclic peptide nanotube by finding the equilibrium distances measured from the tube axis. Similar mathematical techniques presented in Rahmat et al. (2011) and Baowan et al. (2008) are also adopted to determine the explicit formulae of the interaction energies between a molecule and a cyclic peptide nanotube. The results obtained here are consistent with experiments and molecular dynamics simulations of Song et al. (2013), Liu 
et al. (2010), Engels et al. (1995) indicating the arrangement of water molecules as a $1-2-1-2$ file inside the peptide nanotube.

This paper is structured as follows. In Section "Encapsulation of a water molecule in a cyclic peptide nanotube", we determine the energy profile for a water molecule accepting into a peptide nanotube. In Section "An offset water molecule inside a cyclic peptide nanotube", we consider three positions inside the nanotube and at each position we determine the preferred radial position of the water molecule from the nanotube axis. In Section "Interaction of two water molecules inside a cyclic peptide nanotube", we study the interaction between two water molecules and determine the possibility of water molecules forming a $1-2-1-2$ file inside the nanotubes. Finally, we discuss our results and give conclusions in Section "Concluding remarks".

\section{Encapsulation of a water molecule in a cyclic peptide nanotube}

To determine the encapsulation behaviour of a water molecule in a cyclic peptide nanotube, we assume the configuration of our problem as shown in Fig. 1, where a water molecule is assumed to be a combination of a single oxygen atom and a sphere of two hydrogen atoms of radius $b=0.958 \AA$. We note that this radius is based on the $\mathrm{H}-\mathrm{O}$ bond length. A unit of a peptide nanotube is assumed to comprise an inner and an outer tube and each unit stacks to form a stable peptide nanotube. Here, we study the simplest structure of a cyclic peptide nanotube which is the cyclo[(D-Ala-L-Ala) 4- $^{-}$. Based on our assumptions, the interaction between a water molecule and one unit of the cyclic peptide nanotube can be obtained by summing the four interaction energies, namely

$E_{\text {tot }}=E_{\text {atom-inner }}+E_{\text {atom-outer }}+E_{\text {sphere-inner }}+E_{\text {sphere-outer }}$.

Here, $E_{\text {atom-inner }}$ and $E_{\text {atom-outer }}$ denote the interaction energies between a single oxygen atom at the centre of a water molecule and the inner tube and the outer tubes of the cyclic peptide unit, respectively, while $E_{\text {sphere-inner }}$ and $E_{\text {sphere-outer }}$ are the interaction energies between a sphere of two hydrogen atoms and the inner tube and the outer tubes of the cyclic peptide unit, respectively. Assuming atoms on the surface of a molecule are evenly distributed, the interaction energy between two non-bonded molecules can be given by

$E=\eta_{1} \eta_{2} \int_{\Sigma_{2}} \int_{\Sigma_{1}} \Phi(\rho) d \Sigma_{1} d \Sigma_{2}$,

where $\eta_{1}$ and $\eta_{2}$ are the constant atomic densities of the two interacting molecules and $\rho$ is the distance between two typical points on the surfaces $\Sigma_{1}$ and $\Sigma_{2}$. This continuum assumption is appropriate for modelling molecular structures whose surfaces are well defined with evenly distributed atoms. Moreover, this approach significantly reduces calculation time compared to intensive computational methods, such as molecular dynamics simulations.

As mentioned in the "Introduction", we use the Lennard-Jones potential as a force field to determine the interaction energy between two molecules. The LennardJones potential is given by

$\Phi(\rho)=-\frac{A}{\rho^{6}}+\frac{B}{\rho^{12}}$,

where $A$ and $B$ are the attractive and repulsive constants, respectively. We can calculate the constants $A$ and $B$ for the

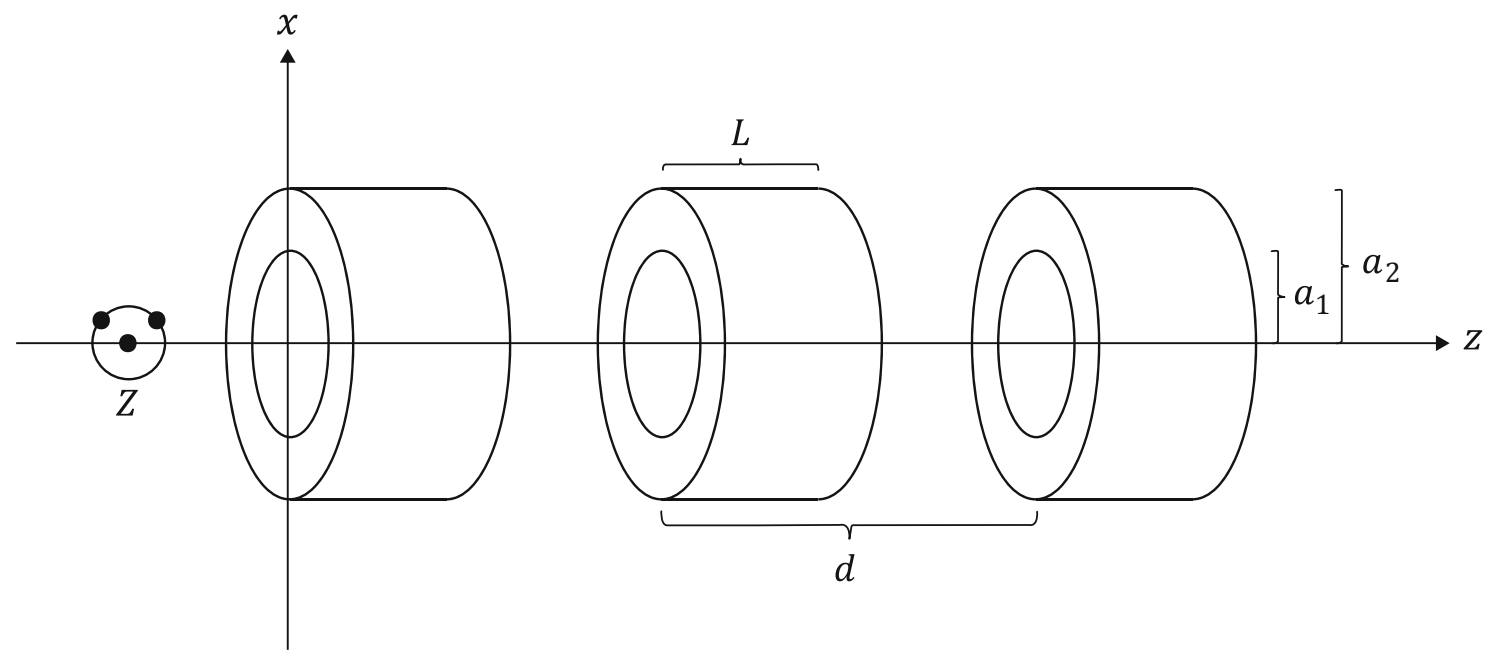

Fig. 1 Schematic model for the interaction between a water molecule and a peptide nanotube comprising three cyclic peptide units 
Table 1 Numerical values of constants used in the model

\begin{tabular}{lll}
\hline Interaction & $A\left(\mathrm{kcal} \mathrm{mol}^{-1} \times \AA^{6}\right)$ & $B\left(\mathrm{kcal} \mathrm{mol}^{-1} \times \AA^{12}\right)$ \\
\hline $\mathrm{C}-\mathrm{H}$ & 198.60 & $1.45 \times 10^{5}$ \\
$\mathrm{C}-\mathrm{O}$ & 391.38 & $4.82 \times 10^{5}$ \\
$\mathrm{C}-\mathrm{C}$ & 684.95 & $1.12 \times 10^{6}$ \\
$\mathrm{H}-\mathrm{H}$ & 50.85 & $1.47 \times 10^{4}$ \\
$\mathrm{H}-\mathrm{N}$ & 135.47 & $8.32 \times 10^{4}$ \\
$\mathrm{H}-\mathrm{O}$ & 108.90 & $5.77 \times 10^{4}$ \\
$\mathrm{~N}-\mathrm{O}$ & 270.91 & $2.85 \times 10^{5}$ \\
$\mathrm{O}-\mathrm{O}$ & 220.59 & $2.03 \times 10^{5}$ \\
\hline
\end{tabular}

interaction between atoms $i$ and $j$ using the formulae $A_{i j}=$ $4 \epsilon_{i j} \sigma_{i j}^{6}$ and $B_{i j}=4 \epsilon_{i j} \sigma_{i j}^{12}$, where $\sigma_{i j}=\left(\sigma_{i}+\sigma_{j}\right) / 2$ and $\epsilon_{i j}=\left(\epsilon_{i} \epsilon_{j}\right)^{1 / 2}$. The numerical values for the energy well depth $\epsilon$ and the van der Waals diameter $\sigma$ are taken from Rappe et al. (1992) and are given in Table 1. It has been shown in Baowan et al. (2013) that the Coulombic potential plays minor role for the energy contribution in the biomolecular system, therefore, only the van der Waals potential is considered here.

From Tiangtrong and Baowan (2014), the interaction between a single atom located at $(0, Z)$ and a nanotube of finite length $L$ is given by

$$
E_{\text {atom-tube }}=2 \pi a \mu_{t}\left(-A I_{3}+B I_{6}\right),
$$

where

$$
\begin{aligned}
I_{n}=a^{1-2 n}\left\{\frac{L-Z}{\left[a^{2}+(L-Z)^{2}\right]^{\frac{1}{2}}} F\left(\frac{3}{2}-n, \frac{1}{2} ; \frac{3}{2} ; \frac{(L-Z)^{2}}{a^{2}+(L-Z)^{2}}\right)\right. \\
\left.+\frac{Z}{\left(a^{2}+Z^{2}\right)^{\frac{1}{2}}} F\left(\frac{3}{2}-n, \frac{1}{2} ; \frac{3}{2} ; \frac{Z^{2}}{a^{2}+Z^{2}}\right)\right\},
\end{aligned}
$$

and $a$ is the radius of the tube. The mean surface density of atoms on the nanotube is denoted by $\mu_{t}$, and $F(\alpha, \beta ; \gamma ; z)$ is a hypergeometric function. We note from Cheng et al. (2009) that the radius of the inner tube $a_{1}$ is $4.25 \AA$, and that of the outer tube $a_{2}$ is $7.95 \AA$. The length of a cyclic peptide unit $L$ is $1.7 \AA$, and the distance between each cyclic peptide unit $d$ is $4.8 \AA$.

From Tiangtrong and Baowan (2014), the interaction between a sphere of radius $b$ and a nanotube of finite length $L$ is given by

$$
\begin{aligned}
E_{\text {sphere-tube }} & =-\frac{8 \pi^{2} a \mu_{t} \mu_{s}}{b^{4}}\left[A\left(K_{3}+2 K_{4}\right)\right. \\
& \left.-\frac{B}{5 b^{6}}\left(5 K_{6}+80 K_{7}+336 K_{8}+512 K_{9}+256 K_{10}\right)\right],
\end{aligned}
$$

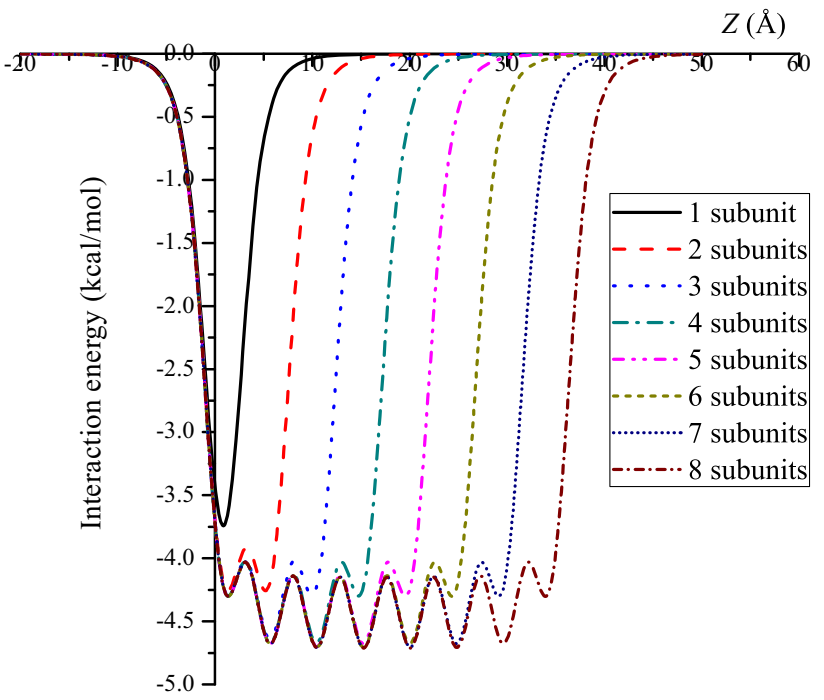

Fig. 2 Interaction energy between a water molecule and cyclo[(-DAla-L-Ala) ${ }_{4}$-] peptide nanotubes

where

$K_{n}=b^{2 n}\left(a^{2}-b^{2}\right)^{\frac{1}{2}-n} \int_{\alpha_{1}}^{\alpha_{2}} \cos ^{2 n-2} \alpha \mathrm{d} \alpha$,

$\mu_{s}$ is the mean surface density of atoms on the sphere, $\alpha_{1}=\arctan \left(\frac{-Z}{\sqrt{a^{2}-b^{2}}}\right) \quad$ and $\quad \alpha_{2}=\arctan \left(\frac{L-Z}{\sqrt{a^{2}-b^{2}}}\right)$. From Gradshteyn and Ryzhik (2000), we have

$$
\int \cos ^{2 p} \varphi \mathrm{d} \varphi=\frac{1}{2^{2 p}}\left\{\left(\begin{array}{c}
2 p \\
p
\end{array}\right) \varphi+\sum_{l=0}^{p-1}\left(\begin{array}{c}
2 p \\
l
\end{array}\right) \frac{\sin [2(p-l) \varphi]}{p-l}\right\},
$$

which enables $K_{n}$ to be determined analytically.

From (1), (2) and (3), we can determine the interaction energy between a water molecule and a cyclic peptide nanotube comprising a finite number of cyclic peptide units. We note that $\mu_{s}=2 /\left(4 \pi b^{2}\right)=0.1734 \AA^{-2}$ for the sphere of two hydrogen atoms. For a cyclo[(-D-Ala-L-

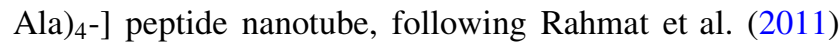
we assume that the inner tube comprises 48 atoms, namely 16 carbon atoms, 16 hydrogen atoms, 8 nitrogen atoms and 8 oxygen atoms, and that the outer tube comprises 32 atoms, namely 8 carbon atoms and 24 hydrogen atoms. Since the atomic surface density for a nanotube is given by $\mu_{t}=$ number of atoms $/(2 \pi a L)$, we have $\mu_{t}=1.0574$ $\AA^{-2}$ and $0.3768 \AA^{-2}$ for the inner and outer tubes, respectively. In Fig. 2, we consider this interaction for cyclic peptide nanotubes comprising one to eight units. From the figure, we can see that a water molecule is encapsulated in the cyclo[(-D-Ala-L-Ala $)_{4}$ - $]$ peptide nanotubes as the energy inside the tube is lower than that externally. We also find that the local minimum energy occurs at the space 
between peptide units implying that the water molecule prefers to be in the space between any two cyclic peptide units. This result agrees with the finding of Engels et al. (1995).

\section{An offset water molecule inside a cyclic peptide nanotube}

As the radius of a water molecule is smaller than that of the nanotube, upon encapsulation into the nanotube, we anticipate that instead of being at the centre of the nanotube, the water molecule prefers to be off-axis, closer to the internal surface of the nanotube to optimise the interaction energy. The off-axis arrangement allows more water molecules to be packed inside the nanotube, i.e. in spiral or zigzag configurations. Here, we investigate the behaviour of an offset water molecule inside a cyclic peptide nanotube of two units. The interaction energy between an offset water molecule and a cyclic peptide nanotube is given by

$E_{\mathrm{tot}}^{\mathrm{offset}}=E_{\text {atom-inner }}^{*}+E_{\text {atom-outer }}^{*}+E_{\text {sphere-inner }}^{*}+E_{\text {sphere-outer }}^{*}$,

where the terms $E_{i-j}^{*}$ are similar to those described in (1), but for the offset molecule here.

We suppose that the centre of the offset water molecule is located at $(\varepsilon, Z)$ inside a cyclic peptide nanotube which is depicted in Fig. 3. Firstly, we consider the interaction between the offset oxygen atom located at $(\varepsilon, Z)$ and the nanotube of finite length $L$ and radius $a$. By letting $\rho_{1}$ be the distance between the offset atom and a surface element of the nanotube, we have $\rho_{1}^{2}=a^{2}+\varepsilon^{2}-2 a \varepsilon \cos \theta+$ $(z-Z)^{2}$. Then, we let $\lambda_{1}=\left(a^{2}+\varepsilon^{2}-2 a \varepsilon \cos \theta\right)^{\frac{1}{2}}$, that is,

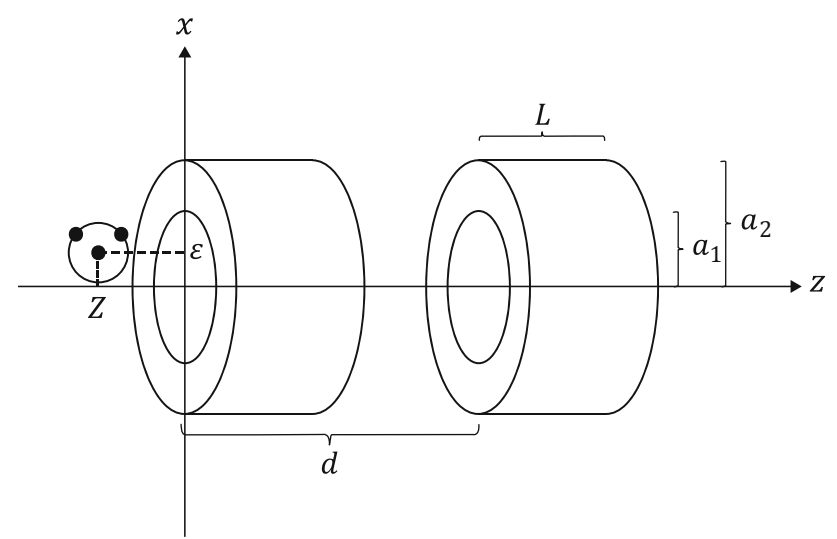

Fig. 3 An offset water molecule inside a cyclic peptide nanotube of two cyclic peptide units
$\rho_{1}^{2}=\lambda_{1}^{2}+(z-Z)^{2}$. Using the continuum approach and the Lennard-Jones potential, the interaction energy is given by

$$
\begin{aligned}
E_{\text {atom-tube }}^{*} & =a \mu_{t} \int_{-\pi}^{\pi} \int_{0}^{L}\left(-\frac{A}{\rho_{1}^{6}}+\frac{B}{\rho_{1}^{12}}\right) \mathrm{d} z \mathrm{~d} \theta \\
& =a \mu_{t} \int_{-\pi}^{\pi} \int_{0}^{L}\left\{-\frac{A}{\left[\lambda_{1}^{2}+(z-Z)^{2}\right]^{3}}+\frac{B}{\left[\lambda_{1}^{2}+(z-Z)^{2}\right]^{6}}\right\} \mathrm{d} z \mathrm{~d} \theta .
\end{aligned}
$$

On substituting $z=Z+\lambda_{1} t$, we have

$$
E_{\text {atom-tube }}^{*}=a \mu_{t} \int_{-\pi}^{\pi} \int_{-\frac{Z}{\lambda_{1}}}^{\frac{L-Z}{\lambda_{1}}}\left[-\frac{A}{\lambda_{1}^{5}\left(1+t^{2}\right)^{3}}+\frac{B}{\lambda_{1}^{11}\left(1+t^{2}\right)^{6}}\right] \mathrm{d} t \mathrm{~d} \theta .
$$

Next, we define

$$
Q_{n}=\int_{-\pi}^{\pi} \int_{-\frac{Z}{\lambda_{1}}}^{\frac{L-Z}{\lambda_{1}}} \frac{\lambda_{1}^{1-2 n}}{\left(1+t^{2}\right)^{n}} \mathrm{~d} t \mathrm{~d} \theta=\int_{-\pi}^{\pi} \lambda_{1}^{1-2 n} \int_{-\frac{Z}{\lambda_{1}}}^{\frac{L-Z}{\lambda_{1}}} \frac{1}{\left(1+t^{2}\right)^{n}} \mathrm{~d} t \mathrm{~d} \theta,
$$

so that

$$
E_{\text {atom-tube }}^{\text {offset }}=a \mu_{t}\left(-A Q_{3}+B Q_{6}\right) \text {. }
$$

Analytical expressions for $Q_{n}$ and full detailed derivation are given in Appendix A.

Using (7), we plot the interaction energy between a cyclic peptide nanotube and an offset oxygen atom, which is assumed to be located at three different positions, $Z=0$, $Z=L / 2$, and $Z=(L+d) / 2$, as shown in Fig. $4 \mathrm{a}, \mathrm{b}, \mathrm{c}$, respectively. These three positions are chosen to represent three scenarios: (i) $Z=0$, an atom is at the extremity of a peptide unit, (ii) $Z=L / 2$, an atom is inside the peptide unit, and (iii) $Z=(L+d) / 2$, an atom is in the space between two peptide units. We comment that at positions (i) and (iii), there is no internal boundary to prevent an atom or a molecule from moving across the radius of the inner nanotube and embedding in the region between the radii of the inner and the outer tubes. However, the atom or the molecule cannot escape the tube through the spaces between the peptide units due to strong hydrogen bonding which dominates the interspacing between cyclic peptide units (Cheng et al. 2009).

From Fig. 4a, b, c, we find that the equilibrium distance $\varepsilon$ in the radial direction measured from the tube axis for the oxygen atom at the three positions is $0.754,0.678$, and $1.382 \AA$, respectively. For all positions, the atom prefers to be off-axis, but still within the internal space of the inner tube.

Next, we consider the interaction between an offset sphere of the hydrogen atoms and a cyclic peptide nanotube comprising two cyclic peptide units, as shown in Fig. 3. We assume that the offset sphere is centred at $(\varepsilon, Z)$. Following Cox et al. (2007), the interaction energy 
(a)

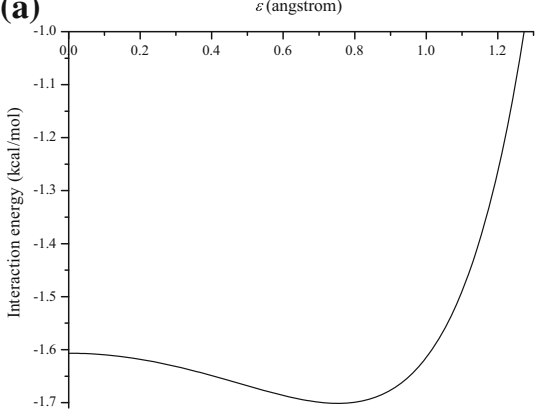

(b)

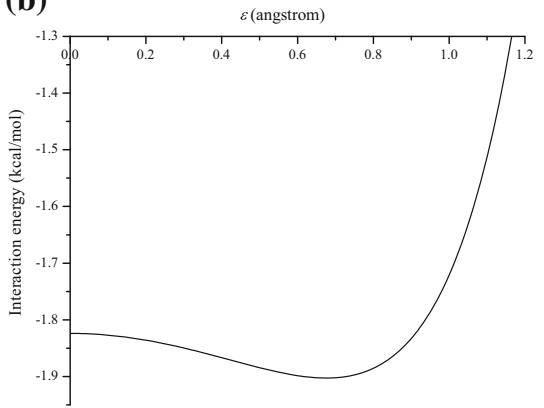

(c)

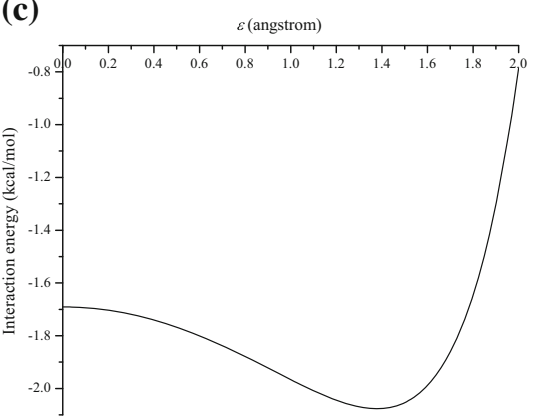

Fig. 4 The interaction energy between an offset atom and a cyclo[(D-Ala-L-Ala) $4_{4}$-] peptide nanotube comprising two cyclic peptide units. The energy profile is plotted with respect to the offset distance $\varepsilon$ from the tube axis and (a), (b), and (c) correspond to the locations $Z=0, L / 2$, and $(L+d) / 2$ of the atom along the tube axis, respectively between a sphere of radius $b$ and a typical atom on the surface of a nanotube of radius $a$ is given by

$$
\begin{aligned}
P(\tilde{\rho})= & -4 \mu_{s} \pi b^{2} A\left(\frac{1}{\left(\tilde{\rho}^{2}-b^{2}\right)^{3}}+\frac{2 b^{2}}{\left(\tilde{\rho}^{2}-b^{2}\right)^{4}}\right) \\
& +\frac{4}{5} \mu_{s} \pi b^{2} B\left(\frac{5}{\left(\tilde{\rho}^{2}-b^{2}\right)^{6}}+\frac{80 b^{2}}{\left(\tilde{\rho}^{2}-b^{2}\right)^{7}}+\frac{336 b^{4}}{\left(\tilde{\rho}^{2}-b^{2}\right)^{8}}\right. \\
& \left.+\frac{512 b^{6}}{\left(\tilde{\rho}^{2}-b^{2}\right)^{9}}+\frac{256 b^{8}}{\left(\tilde{\rho}^{2}-b^{2}\right)^{10}}\right),
\end{aligned}
$$

where $\tilde{\rho}$ is the distance between the centre of the sphere and a typical atom of the nanotube surface, and $\tilde{\rho}=\left[a^{2}-2 a \varepsilon \cos \theta+\varepsilon^{2}+(z-Z)^{2}\right]^{\frac{1}{2}}$. Therefore, the interaction energy between an offset sphere and the nanotube of finite length $L$ is given by

$E_{\text {sphere-tube }}^{*}=a \mu_{t} \int_{-\pi}^{\pi} \int_{0}^{L} P(\tilde{\rho}) \mathrm{d} z \mathrm{~d} \theta$.

Let $\lambda_{2}^{2}=a^{2}-2 a \varepsilon \cos \theta+\varepsilon^{2}-b^{2}$, we have

$$
\begin{aligned}
P & =-4 \mu_{s} \pi b^{2} A\left\{\frac{1}{\left[\lambda_{2}^{2}+(z-Z)^{2}\right]^{3}}+\frac{2 b^{2}}{\left[\lambda_{2}^{2}+(z-Z)^{2}\right]^{4}}\right\}+\frac{4}{5} \mu_{s} \pi b^{2} B \\
& \times\left\{\frac{5}{\left[\lambda_{2}^{2}+(z-Z)^{2}\right]^{6}}+\frac{80 b^{2}}{\left[\lambda_{2}^{2}+(z-Z)^{2}\right]^{7}}+\frac{336 b^{4}}{\left[\lambda_{2}^{2}+(z-Z)^{2}\right]^{8}}\right. \\
& \left.+\frac{512 b^{6}}{\left[\lambda_{2}^{2}+(z-Z)^{2}\right]^{9}}+\frac{256 b^{8}}{\left[\lambda_{2}^{2}+(z-Z)^{2}\right]^{10}}\right\} .
\end{aligned}
$$

We make a substitution $z=Z+\lambda_{2} t$ to obtain

$$
\begin{aligned}
P= & -4 \mu_{s} \pi b^{2} A\left[\frac{1}{\lambda_{2}^{6}\left(1+t^{2}\right)^{3}}+\frac{2 b^{2}}{\lambda_{2}^{8}\left(1+t^{2}\right)^{4}}\right] \\
+\frac{4}{5} \mu_{s} \pi b^{2} B & {\left[\frac{5}{\lambda_{2}^{12}\left(1+t^{2}\right)^{6}}\right.} \\
& +\frac{80 b^{2}}{\lambda_{2}^{14}\left(1+t^{2}\right)^{7}}+\frac{336 b^{4}}{\lambda_{2}^{16}\left(1+t^{2}\right)^{8}} \\
& \left.+\frac{512 b^{6}}{\lambda_{2}^{18}\left(1+t^{2}\right)^{9}}+\frac{256 b^{8}}{\lambda_{2}^{20}\left(1+t^{2}\right)^{10}}\right] .
\end{aligned}
$$

Then, we let

$T_{n}=\int_{-\pi}^{\pi} \int_{-\frac{Z}{\lambda_{2}}}^{\frac{L-Z}{\lambda_{2}}} \frac{\lambda_{2}^{1-2 n}}{\left(1+t^{2}\right)^{n}} \mathrm{~d} t \mathrm{~d} \theta$.

From (8), (9) and (10), we deduce

$$
\begin{aligned}
E_{\text {sphere-tube }}^{*}=a & \mu_{t}\left[-4 \mu_{s} \pi b^{2} A\left(T_{3}+2 b^{2} T_{4}\right)\right. \\
& +\frac{4}{5} \mu_{s} \pi b^{2} B\left(5 T_{6}+80 b^{2} T_{7}\right. \\
& \left.\left.+336 b^{4} T_{8}+512 b^{6} T_{9}+256 b^{8} T_{10}\right)\right] .
\end{aligned}
$$

We comment that $Q_{n}$ defined by (6) is equivalent to $T_{n}$ in (10) where $\lambda_{1}$ is replaced by $\lambda_{2}$. Therefore, for any given integer $n$, we can determine an analytical expression for $T_{n}$, as given in Appendix B.

Figure $5 \mathrm{a}, \mathrm{b}, \mathrm{c}$ shows the interaction between the cyclo[(-D-Ala-L-Ala $)_{4}$ - peptide nanotube comprising two units and an offset sphere of two hydrogen atoms of radius $b=0.958 \AA$, assumed to be located at three positions $Z=0, Z=L / 2$, and $Z=(L+d) / 2$, respectively. The equilibrium distance $\varepsilon$ for the sphere at the three positions is $0.305,0.151$, and $1.000 \AA$, respectively. For all three cases, the sphere prefers to be off-axis, but is still within 
(a)

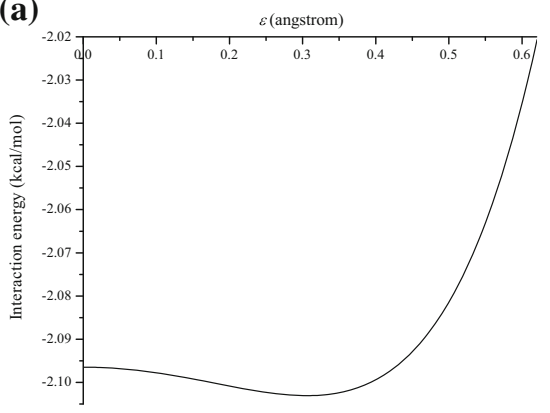

(b)

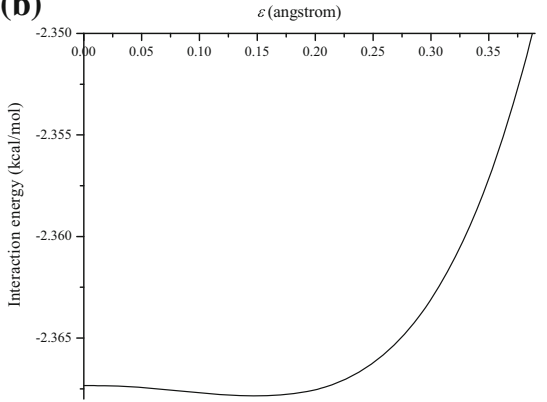

(c)

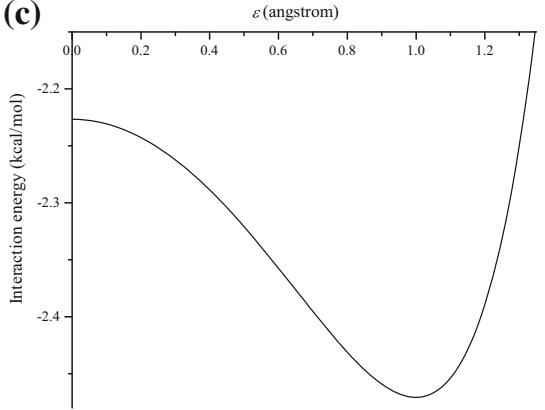

Fig. 5 The interaction energy between an offset sphere and a cyclo[(-D-Ala-L-Ala) $\left.)_{4}-\right]$ peptide nanotube comprising two cyclic peptide units. The energy profile is plotted with respect to the offset distance $\varepsilon$ from the tube axis and (a), (b), and (c) correspond to the locations $Z=0, L / 2$, and $(L+d) / 2$ of the sphere along the tube axis, respectively (a)

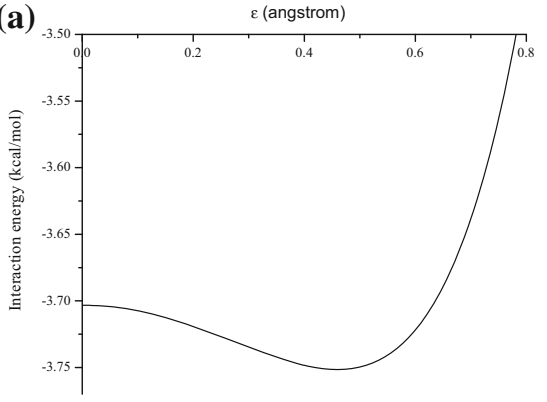

(b)

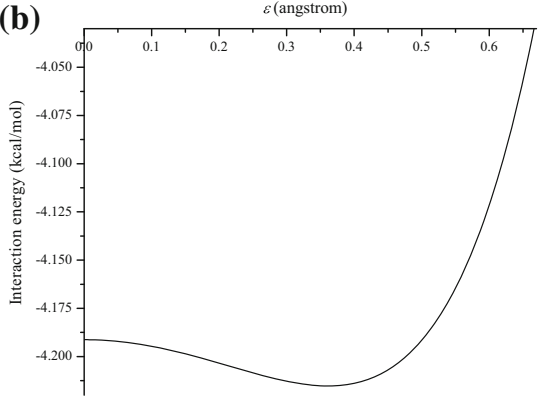

(c)

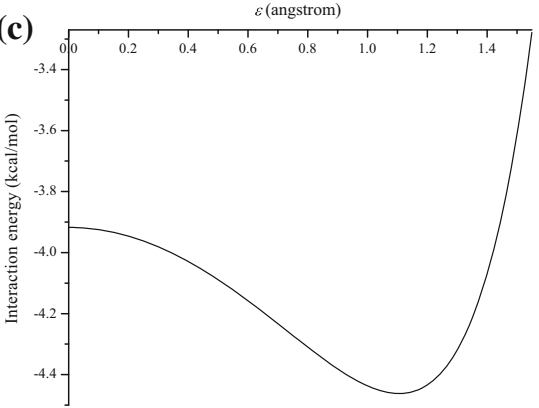

Fig. 6 The interaction energy between an offset water molecule and a cyclo[(-D-Ala-L-Ala) $4_{4}$-] peptide nanotube comprising two cyclic peptide units. The energy profile is plotted with respect to the offset distance $\varepsilon$ from the tube axis and (a), (b), and (c) correspond to the locations $Z=0, L / 2$, and $(L+d) / 2$ of the water molecule along the tube axis, respectively the internal area of the inner tube. Note that, the off-axis distance for the case $Z=L / 2$ is smallest due to the space restriction between the inner nanotube's wall and the sphere.

Finally, we investigate the interactions between the cyclo[(-D-Ala-L-Ala $\left.)_{4}-\right]$ peptide nanotube and an offset water molecule using (5), (7) and (11). Again, we assume three positions for the water molecule which are $Z=0$, $Z=L / 2$, and $Z=(L+d) / 2$. Corresponding results are shown in Fig. 6a, b, c, for the three locations of the water molecule, namely (i) at the extremity of tube, (ii) inside the peptide unit, and (iii) in the space between two peptide units, respectively. From the figures, the equilibrium position $\varepsilon$ for the water molecule at the three positions is $0.459,0.360$, and $1.102 \AA$, respectively. These results suggest that the water molecule prefers to be within the internal space of the inner tube, but at an offset distance away from the central axis of the nanotube.

In the following section, we consider the possibility of having two water molecules located at each of these three positions. We note for the position (ii) that the closest distance between the surface of the water molecule and the inner boundary is $d=2.932 \AA$. If the water molecule is closer than this distance, it will become repelled meaning that the system is not at equilibrium.

\section{Interaction of two water molecules inside a cyclic peptide nanotube}

Here, we consider if two water molecules can be in the same cross-sectional plane inside the peptide nanotube (see Fig. 7). By considering this, we are able to predict whether the equilibrium arrangement of the water molecules inside the peptide nanotube will be a single file as seen in Raghavender et al. $(2009,2010)$ or a $1-2-1-2$ file as suggested by Engels et al. (1995), Song et al. (2013), Liu et al. (2010). In this section, we assume that the peptide nanotube only comprises two peptide units.

We first consider the interaction energy between two water molecules centred at a distance $\rho$ apart, given by

$$
\begin{aligned}
E_{\text {water-water }}(\rho)= & E_{\text {atom-atom }}(\rho)+2 E_{\text {atom-sphere }}(\rho) \\
& +E_{\text {sphere-sphere }}(\rho),
\end{aligned}
$$

where $E_{\text {atom-atom }}$ is the energy between two oxygen atoms, $E_{\text {atom-sphere }}$ is the energy between an oxygen atom and a 


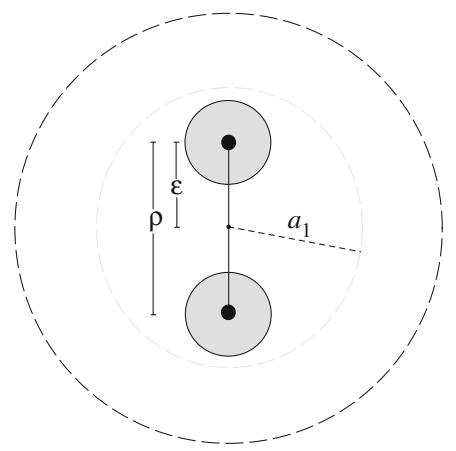

Fig. 7 Schematic model for the interaction between two water molecules at the same cross-sectional plane in the space between two peptide units

sphere of two hydrogen atoms and $E_{\text {sphere-sphere }}$ is the energy between two spheres of hydrogen atoms. These interaction energies are given by

$$
\begin{aligned}
E_{\text {atom-atom }}(\rho)=-\frac{A_{O-O}}{\rho^{6}}+\frac{B_{O-O}}{\rho^{12}}, & \\
E_{\text {atom-sphere }}(\rho)=\frac{2 \pi b \mu_{s}}{\rho} & {\left[\frac{A_{H-O}}{2}\left(\frac{1}{(\rho+b)^{4}}-\frac{1}{(\rho-b)^{4}}\right)\right.} \\
& \left.-\frac{B_{H-O}}{5}\left(\frac{1}{(\rho+b)^{10}}-\frac{1}{(\rho-b)^{10}}\right)\right],
\end{aligned}
$$

$E_{\text {sphere-sphere }}(\rho)=-A_{H-H} J_{6}+B_{H-H} J_{12}$,

where $J_{n}$ is defined by

$$
\begin{aligned}
J_{n}= & \frac{4 \pi^{2} b^{2} \mu_{s}^{2}}{\rho(2-n)(3-n)}\left\{\frac{1}{(2 b+\rho)^{n-3}}+\frac{1}{(-\rho)^{n-3}}\right. \\
& \left.-\frac{1}{(2 b-\rho)^{n-3}}-\frac{1}{\rho^{n-3}}\right\} .
\end{aligned}
$$

The radius of a sphere of hydrogen atoms is $b=0.958 \AA$, $\mu_{s}$ is the mean atomic surface density of the sphere and the values for $A_{i-j}$ and $B_{i-j}$ for each type of interactions are given in Table 1. Derivation of (14) and (15) is given in Thamwattana et al. (2009). In Fig. 8, we plot the energy for the two water molecules as a function of the distance $\rho$ between their centres. From the figure, the energy is minimum when the two water molecules are located apart at the equilibrium distance $\rho_{\min }^{(1)}=3.931 \AA$. Based on Fig. 7, we find that the closest distance between the surface of the water molecule and the inner boundary is $1.327 \AA$. Compared with the value $d=2.932 \AA$ at the position (ii) obtained in the previous section, we can say that two water molecules cannot be accommodated at a cross-sectional plane inside the peptide unit as the water molecules are too

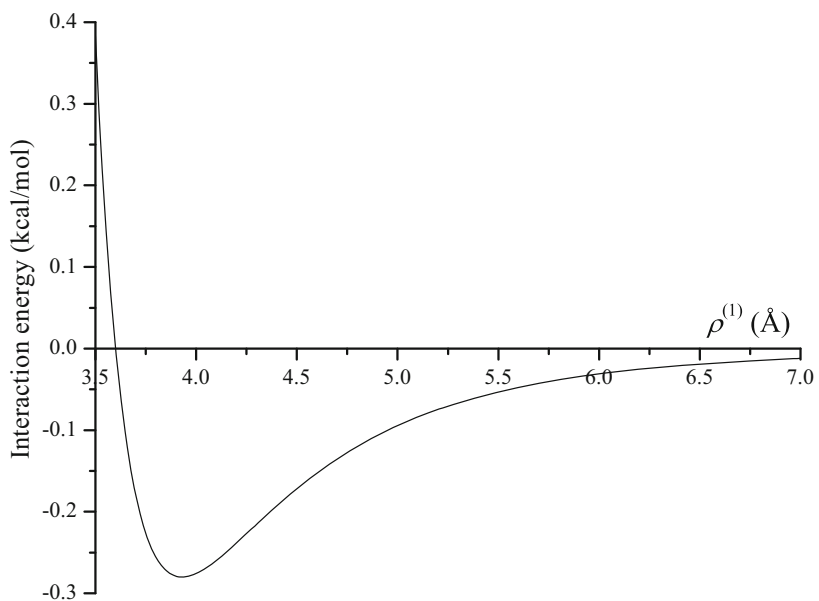

Fig. 8 Interaction energy between two water molecules at a distance $\rho$ apart

close to the surface of the inner nanotube. However, this value is obtained without considering the effect from the inner and the outer nanotubes of the two adjacent peptide units.

To address this shortcoming and confirm our prediction, we add the energy contribution from the inner and the outer nanotubes of the two adjacent peptide units to (12) to find the total interaction energy. We also calculate this for the three positions of the two water molecules, $Z=0$, $Z=L / 2$, and $Z=(L+d) / 2$. The interaction energies corresponding to these three positions are given in Fig. 9a, b, c, respectively. From Fig. 9a when the water molecule is located at the extremity of the nanotube and Fig. $9 b$ when the water molecule is inside the peptide unit, we find that the interaction energies for both cases are positive. These results imply that at these positions, two water molecules cannot be on the same cross-sectional plane simultaneously as the energy is repulsive, giving rise to an unstable system. However, from Fig. 9c, we find that two water molecules can be accommodated simultaneously in the space between the two peptide units with the equilibrium position $\varepsilon=$ $1.681 \AA$. Since $\rho=2 \varepsilon$, the equilibrium distance between the two water molecules is given by $\rho_{\text {min }}^{(2)}=3.362 \AA$, which is in a reasonable agreement with the value of $3.0 \AA$ found in Liu et al. (2010). We comment that the water molecules become closer when considering the effect from the the inner and the outer nanotubes of the two adjacent peptide units. Based on the above results, we have shown that only one water molecule can occupy inside the peptide unit, while there can exist two water molecules simultaneously in the interspacing between the peptide units. This finding agrees with Song et al. (2013), Liu et al. (2010), Engles et al. (1995) confirming that water molecules inside the peptide nanotube form a $1-2-1-2$ file. 

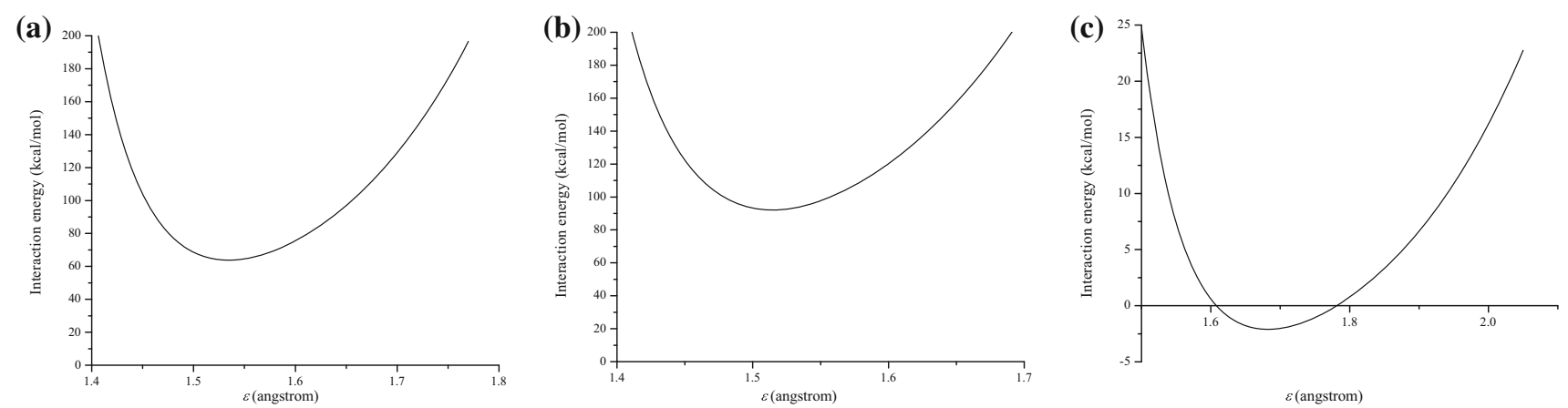

Fig. 9 The interaction energy between two offset water molecules and a cyclo[(-D-Ala-L-Ala) $)_{4}$ - peptide nanotube comprising two cyclic peptide units. The energy profile is plotted with respect to the

\section{Concluding remarks}

This paper studies the interaction energy between a water molecule and a cyclo[(-D-Ala-L-Ala $)_{4}$ - $]$ peptide nanotube comprising a finite number of cyclic peptide units. Our model assumes that each unit of a nanotube comprises an inner and an outer tube and that a water molecule is made up of a sphere on which two hydrogen atoms are uniformly distribute over its surface and a single oxygen atom at the centre of the sphere. We assume the radius of the sphere to be $b=0.958 \AA$, based on the bond length of $\mathrm{H}-\mathrm{O}$ in the water molecule. To find the interaction between a peptide nanotube and a water molecule, we first need to determine the interaction energies for a single atom and the nanotube and for a sphere and the nanotube. Then, by combining the four interaction components, we obtain the total energy of a water molecule inside the cyclic peptide nanotube. We also study cyclic peptide nanotubes comprising one to eight cyclic peptide units; the energy profiles are as shown in Fig. 2. Our results show that a water molecule is accepted into all cyclic peptide nanotubes studied. Moreover, we find that the minimum interaction energy is at the locations corresponding to the spaces between any two cyclic peptide units, indicating that once becoming inside a nanotube, a water molecule will prefer to be in the space between peptide units rather than staying within the internal area of the peptide unit. This result is consistent with that of Engels et al. (1995) and the prefer position of an ion-water cluster inside a peptide nanotube (Rahmat et al. 2011).

For a water molecule inside a peptide nanotube, based on their dimensions, it is predicted that to optimise their interaction the water molecule will be off-axis closer to the surface of the inner nanotube. This paper determines the equilibrium offset distance $\varepsilon$ from the tube axis for the possible three positions of a water molecule inside the tube, namely (i) $Z=0$, the molecule is at the extremity of a peptide unit, (ii) $Z=L / 2$, the molecule is inside the peptide unit, and (iii) $Z=(L+d) / 2$, the molecule is in the offset distance $\varepsilon$ from the tube axis and (a), (b), and (c) correspond to the locations $Z=0, L / 2$, and $(L+d) / 2$ of the two water molecules along the tube axis, respectively

space between two peptide units. We determine analytical expressions for the interaction energy between an offset water molecule and the cyclic peptide nanotube and plot the energy versus the offset distance $\varepsilon$ to find the equilibrium position of the water molecule. Firstly, we determine the interaction energy between the cyclic peptide nanotube and an offset atom and the interaction energy between the cyclic peptide nanotube and an offset spherical molecule of radius $b=0.958 \AA$. Then, we combine the interaction energies to obtain the total interaction energy for an offset water molecule. Our results show that the equilibrium offset distances $\varepsilon$ of a water molecule corresponding to positions (i), (ii), and (iii) are $0.459,0.360$, and $1.102 \AA$, respectively. These findings suggest that a water molecule prefers to be offset from the tube axis for all three positions. The off-axis position in the interspacing between two cyclic peptide units allows two water molecules to be in the same plane. However, due to the space restriction, there can only be one water molecule inside the peptide unit. These findings are in agreement with the results in Song et al. (2013), Liu et al. (2010), Engels et al. (1995) that water molecules arrange in the form of a $1-2-1-2$ file inside a peptide nanotube.

Our findings facilitate an understanding between the interaction of water molecules and peptide nanotubes which could be thought of as the first step toward the investigation of drug or gene encapsulated into peptide nanotubes for biomedical research. Furthermore, it may be beneficial to experimental and molecular dynamics studies reducing the trial-and-error processes.

Acknowledgments The authors acknowledge the financial support from the University of Wollongong's Internationalisation Linkage Grant Scheme. Prangsai Tiangtrong is grateful to the Sri Trung Thong Scholarship, Faculty of Science, Mahidol University, for the provision of a PhD scholarship. The Thailand Research Fund (TRG5680072) is also gratefully acknowledged.

Open Access This article is distributed under the terms of the Creative Commons Attribution License which permits any use, 
distribution, and reproduction in any medium, provided the original author(s) and the source are credited.

\section{A: Derivation of $Q_{n}$ for the interaction energy of an offset atom inside a peptide nanotube}

By substituting $t=\tan \phi$ into (6), we have

$$
Q_{n}=\int_{-\pi}^{\pi} \lambda_{1}^{1-2 n} \int_{\arctan \left(-\frac{Z}{\lambda_{1}}\right)}^{\arctan \left(\frac{L-Z}{\lambda_{1}}\right)} \cos ^{2(n-1)} \phi \mathrm{d} \phi \mathrm{d} \theta .
$$

Now, we can determine $Q_{3}$ which is given by

$$
\begin{aligned}
Q_{3}=\int_{-\pi}^{\pi} & \frac{1}{\lambda_{1}^{5}} \int_{\arctan \left(-\frac{Z}{\lambda_{1}}\right)}^{\arctan \left(\frac{L-Z}{\lambda_{1}}\right)} \cos ^{4} \phi \mathrm{d} \phi \mathrm{d} \theta \\
=\int_{-\pi}^{\pi} & \left\{\frac{3}{8} \frac{\arctan \left(\frac{L-Z}{\lambda_{1}}\right)}{\lambda_{1}^{5}}+\frac{1}{4} \frac{L-Z}{\lambda_{1}^{2}\left[(L-Z)^{2}+\lambda_{1}^{2}\right]^{2}}\right. \\
& +\frac{3}{8} \frac{L-Z}{\lambda_{1}^{4}\left[(L-Z)^{2}+\lambda_{1}^{2}\right]} \\
& \left.-\frac{3}{8} \frac{\arctan \left(-\frac{Z}{\lambda_{1}}\right)}{\lambda_{1}^{5}}+\frac{1}{4} \frac{Z}{\lambda_{1}^{2}\left(Z^{2}+\lambda_{1}^{2}\right)^{2}}+\frac{3}{8} \frac{Z}{\lambda_{1}^{4}\left(Z^{2}+\lambda_{1}^{2}\right)}\right\} \mathrm{d} \theta .
\end{aligned}
$$

Similarly, we calculate $Q_{6}$ which is given by

$$
\begin{aligned}
& Q_{6}=\int_{-\pi}^{\pi} \frac{1}{\lambda_{1}^{11}} \int_{\arctan \left(-\frac{Z}{\lambda_{1}}\right)}^{\arctan \left(\frac{L-Z}{\lambda_{1}}\right)} \cos ^{10} \phi \mathrm{d} \phi \mathrm{d} \theta \\
& =\int_{-\pi}^{\pi}\left\{\frac{63}{256} \frac{\arctan \left(\frac{L-Z}{\lambda_{1}}\right)}{\lambda_{1}^{11}}+\frac{1}{10} \frac{L-Z}{\lambda_{1}^{2}\left[(L-Z)^{2}+\lambda_{1}^{2}\right]^{5}}\right. \\
& +\frac{9}{80} \frac{L-Z}{\lambda_{1}^{4}\left[(L-Z)^{2}+\lambda_{1}^{2}\right]^{4}} \\
& +\frac{21}{160} \frac{L-Z}{\lambda_{1}^{6}\left[(L-Z)^{2}+\lambda_{1}^{2}\right]^{3}}+\frac{21}{128} \frac{L-Z}{\lambda_{1}^{8}\left[(L-Z)^{2}+\lambda_{1}^{2}\right]^{2}} \\
& +\frac{63}{256} \frac{L-Z}{\lambda_{1}^{10}\left[(L-Z)^{2}+\lambda_{1}^{2}\right]} \\
& -\frac{63}{256} \frac{\arctan \left(\frac{-Z}{\lambda_{1}}\right)}{\lambda_{1}^{11}}+\frac{1}{10} \frac{Z}{\lambda_{1}^{2}\left(Z^{2}+\lambda_{1}^{2}\right)^{5}}+\frac{9}{80} \frac{Z}{\lambda_{1}^{4}\left(Z^{2}+\lambda_{1}^{2}\right)^{4}} \\
& +\frac{21}{160} \frac{Z}{\lambda_{1}^{6}\left(Z^{2}+\lambda_{1}^{2}\right)^{3}}+\frac{21}{128} \frac{Z}{\lambda_{1}^{8}\left(Z^{2}+\lambda_{1}^{2}\right)^{2}} \\
& \left.+\frac{63}{256} \frac{Z}{\lambda_{1}^{10}\left(Z^{2}+\lambda_{1}^{2}\right)}\right\} \mathrm{d} \theta \text {. }
\end{aligned}
$$

From (16) and (17), we can see that the following two terms are needed to be evaluated,

$$
\begin{aligned}
& R_{(m, n)}^{(j)}=\int_{-\pi}^{\pi} \frac{1}{\lambda_{1}^{m}\left(\lambda_{1}^{2}+C_{j}^{2}\right)^{n}} \mathrm{~d} \theta, \\
& S_{n}^{(j)}=\int_{-\pi}^{\pi} \frac{\arctan \left(\frac{C_{j}}{\lambda_{1}}\right)}{\lambda_{1}^{n}} d \theta,
\end{aligned}
$$

where $C_{j}$ are constants, $C_{1}=-Z$ and $C_{2}=L-Z$. Here, we evaluate $R_{(m, n)}^{(j)}$ defined by (18). From $\lambda_{1}^{2}=a^{2}+\varepsilon^{2}-2 a \varepsilon \cos \theta$, we substitute $x=\theta / 2$ to get $\lambda_{1}^{2}=(a-\varepsilon)^{2}+4 a \varepsilon \sin ^{2} x$.

Let $\mu=(a-\varepsilon)^{2}, \sigma=(a-\varepsilon)^{2}+C_{j}^{2}$ and $v=4 a \varepsilon$, then we have

$$
R_{(m, n)}^{(j)}=4 \int_{0}^{\frac{\pi}{2}} \frac{1}{\left(\mu+v \sin ^{2} x\right)^{\frac{m}{2}}\left(\sigma+v \sin ^{2} x\right)^{n}} \mathrm{~d} x .
$$

Substitute $t=\cot x$, and let $\beta=\mu /(\mu+v)$ and $\gamma=\sigma /(\sigma+$ v) to obtain

$$
\begin{aligned}
R_{(m, n)}^{(j)} & =4 \int_{0}^{\infty} \frac{\left(1+t^{2}\right)^{\frac{m}{2}+n-1}}{\left(v+\mu+\mu t^{2}\right)^{\frac{m}{2}}\left(v+\sigma+\sigma t^{2}\right)^{n}} d t \\
= & \frac{4}{(\mu+v)^{\frac{m}{2}}(\sigma+v)^{n}} \\
& \times \int_{0}^{\infty} \frac{1}{\left[1-(1-\beta) t^{2} /\left(1+t^{2}\right)\right]^{\frac{m}{2}}\left[1-(1-\gamma) t^{2} /\left(1+t^{2}\right)\right]^{n}\left(1+t^{2}\right)} d t .
\end{aligned}
$$

Next, we substitute $z=\frac{t}{\left(1+t^{2}\right)^{\frac{1}{2}}}$, that is, $t=\frac{z}{\left(1-z^{2}\right)^{\frac{1}{2}}}$, we may deduce

$$
R_{(m, n)}^{(j)}=\frac{4}{(\mu+v)^{\frac{m}{2}}(\sigma+v)^{n}} \int_{0}^{1} \frac{\left(1-z^{2}\right)^{-\frac{1}{2}}}{\left[1-(1-\beta) z^{2}\right]^{\frac{m}{2}}\left[1-(1-\gamma) z^{2}\right]^{n}} \mathrm{~d} z .
$$

Then, we substitute $u=z^{2}$ to obtain

$$
R_{(m, n)}^{(j)}=\frac{2}{(\mu+v)^{\frac{m}{2}}(\sigma+v)^{n}} \int_{0}^{1} \frac{u^{-\frac{1}{2}}(1-u)^{-\frac{1}{2}}}{[1-(1-\beta) u]^{\frac{m}{2}}[1-(1-\gamma) u]^{n}} \mathrm{~d} u .
$$

An Appell hypergeometric function of two variables and of the first kind is defined in Bailey (1972), namely

$$
F_{1}\left(\alpha ; \beta, \beta^{\prime} ; \gamma ; x, y\right)=\sum_{n=0}^{\infty} \sum_{m=0}^{\infty} \frac{(\alpha)_{m+n}(\beta)_{m}\left(\beta^{\prime}\right)_{n}}{m ! n !(\gamma)_{m+n}} x^{m} y^{n} .
$$

Moreover, we have

$$
\frac{\Gamma(\alpha) \Gamma(\gamma-\alpha)}{\Gamma(\gamma)} F_{1}\left(\alpha ; \beta, \beta^{\prime} ; \gamma ; x, y\right)=\int_{0}^{1} \frac{u^{\alpha-1}(1-u)^{\gamma-\alpha-1}}{(1-u x)^{\beta}(1-u y)^{\beta^{\prime}}} \mathrm{d} u,
$$

where $\Gamma(\alpha)$ is a gamma function and

$$
F_{1}\left(\alpha ; \beta, \beta^{\prime} ; \gamma ; x, y\right)=\sum_{i=0}^{\infty} \frac{(\alpha)_{i}(\beta)_{i}}{i !(\gamma)_{i}} F\left(\alpha+i, \beta^{\prime} ; \gamma+i ; y\right) x^{i} .
$$

Therefore, the closed form of $R_{(m, n)}^{(j)}$ is 


$$
\begin{aligned}
R_{(m, n)}^{(j)}= & \frac{2 \pi}{(a+\varepsilon)^{m}\left[(a+\varepsilon)^{2}+C_{j}^{2}\right]^{n}} \sum_{i=0}^{\infty} \frac{(1 / 2)_{i}(m / 2)_{i}}{(i !)^{2}} \\
& \times F\left(\frac{1}{2}+i, n ; 1+i ; \frac{4 a \varepsilon}{(a+\varepsilon)^{2}+C_{j}^{2}}\right)\left[\frac{4 a \varepsilon}{(a+\varepsilon)^{2}}\right]^{i} .
\end{aligned}
$$

We note that $\mu+v=(a+\varepsilon)^{2}, \sigma+v=(a+\varepsilon)^{2}+C_{j}^{2}, \beta=$ $(a-\varepsilon)^{2} /(a+\varepsilon)^{2}, \quad$ and $\quad \gamma=\left[(a-\varepsilon)^{2}+C_{j}^{2}\right] /\left[(a+\varepsilon)^{2}\right.$ $\left.+C_{j}^{2}\right]$. Next, we determine $S_{n}^{(j)}$ defined by (19), and using the same substitution $x=\theta / 2$, we get

$S_{n}^{(j)}=4 \int_{0}^{\frac{\pi}{2}} \frac{\arctan \left(\frac{C_{j}}{\lambda_{1}}\right)}{\lambda_{1}^{n}} \mathrm{~d} x$

Similarly, we let $\mu=(a-\varepsilon)^{2}, \sigma=(a-\varepsilon)^{2}+C_{j}^{2}$, and $v=$ $4 a \varepsilon$ to have $\lambda_{1}^{2}=\mu+v \sin ^{2} x$. Since $\left(\frac{C_{j}}{\lambda_{1}}\right)^{2}$ is well defined, we have

$$
\arctan \left(\frac{C_{j}}{\lambda_{1}}\right)=\frac{C_{j}}{\sqrt{\lambda_{1}^{2}+C_{j}^{2}}} \sum_{k=0}^{\infty} \frac{(2 k) !}{2^{2 k}(k !)^{2}(2 k+1)}\left(\frac{C_{j}^{2}}{\lambda_{1}^{2}+C_{j}^{2}}\right)^{k} .
$$

Therefore, we may deduce

$$
\begin{aligned}
S_{n}^{(j)} & =4 \int_{0}^{\frac{\pi}{2}} \frac{1}{\lambda_{1}^{n}}\left[\frac{C_{j}}{\sqrt{\lambda_{1}^{2}+C_{j}^{2}}} \sum_{k=0}^{\infty} \frac{(2 k) !}{2^{2 k}(k !)^{2}(2 k+1)}\left(\frac{C_{j}^{2}}{\lambda_{1}^{2}+C_{j}^{2}}\right)^{k}\right] \mathrm{d} x \\
& =\sum_{k=0}^{\infty} \frac{C_{j}^{2 k+1}(2 k) !}{2^{2 k-2}(k !)^{2}(2 k+1)} \int_{0}^{\frac{\pi}{2}} \frac{1}{\lambda_{1}^{n}\left(\lambda_{1}^{2}+C_{j}^{2}\right)^{k+\frac{1}{2}}} \mathrm{~d} x .
\end{aligned}
$$

From the evaluation of $R_{(m, n)}^{(j)}$, the closed form of $S_{n}^{(j)}$ is

$$
\begin{aligned}
S_{n}^{(j)}= & \pi \sum_{k=0}^{\infty} \sum_{i=0}^{\infty} \frac{C_{j}^{2 k+1}(2 k) !}{2^{2 k-1}(k !)^{2}(2 k+1)(a+\varepsilon)^{n}\left[(a+\varepsilon)^{2}+C_{j}^{2}\right]^{k+\frac{1}{2}}} \frac{(1 / 2)_{i}(n / 2)_{i}}{(i !)^{2}} \\
& \times F\left(\frac{1}{2}+i, k+\frac{1}{2} ; 1+i ; \frac{4 a \varepsilon}{(a+\varepsilon)^{2}+C_{j}^{2}}\right)\left[\frac{4 a \varepsilon}{(a+\varepsilon)^{2}}\right]^{i}
\end{aligned}
$$

From (16), (17), (20), and (21), we write $Q_{3}$ and $Q_{6}$ in terms of $R_{(m, n)}^{(j)}$ and $S_{n}^{(j)}$ as

$$
\begin{aligned}
Q_{3}= & \frac{3}{8} S_{5}^{(2)}+\frac{1}{4} C_{2} R_{(2,2)}^{(2)}+\frac{3}{8} C_{2} R_{(4,1)}^{(2)}-\frac{3}{8} S_{5}^{(1)}-\frac{1}{4} C_{1} R_{(2,2)}^{(1)}-\frac{3}{8} C_{1} R_{(4,1)}^{(1)}, \\
Q_{6}= & \frac{63}{256} S_{11}^{(2)}+\frac{1}{10} C_{2} R_{(2,5)}^{(2)}+\frac{9}{80} C_{2} R_{(4,4)}^{(2)}+\frac{21}{160} C_{2} R_{(6,3)}^{(2)}+\frac{21}{128} C_{2} R_{(8,2)}^{(2)} \\
& +\frac{63}{256} C_{2} R_{(10,1)}^{(2)}-\frac{63}{256} S_{11}^{(1)}-\frac{1}{10} C_{1} R_{(2,5)}^{(1)}-\frac{9}{80} C_{1} R_{(4,4)}^{(1)}-\frac{21}{160} C_{1} R_{(6,3)}^{(1)} \\
& -\frac{21}{128} C_{1} R_{(8,2)}^{(1)}-\frac{63}{256} C_{1} R_{(10,1)}^{(1)} .
\end{aligned}
$$

Upon the substitution of $Q_{3}$ and $Q_{6}$ into (7), we can obtain the interaction energy between an offset atom inside a nanotube.

\section{B: Derivation of $T_{n}$ for the interaction energy of an offset sphere inside a peptide nanotube}

Following the same technique described for the evaluation of $Q_{n}$, there are two remaining integrations which are needed to be determined, namely

$$
\begin{aligned}
& U_{(m, n)}^{(j)}=\int_{-\pi}^{\pi} \frac{1}{\lambda_{2}^{m}\left(\lambda_{2}^{2}+C_{j}^{2}\right)^{n}} \mathrm{~d} \theta, \\
& V_{n}^{(j)}=\int_{-\pi}^{\pi} \frac{\arctan \left(\frac{C_{j}}{\lambda_{2}}\right)}{\lambda_{2}^{n}} \mathrm{~d} \theta .
\end{aligned}
$$

Using the similar method to obtain the closed forms of $R_{(m, n)}^{(j)}$ and $S_{n}^{(j)}$, we find

$$
\begin{aligned}
U_{(m, n)}^{(j)}= & \frac{2 \pi}{\left[(a+\varepsilon)^{2}-b^{2}\right]^{\frac{m}{2}}\left[(a+\varepsilon)^{2}+C_{j}^{2}-b^{2}\right]^{n}} \sum_{i=0}^{\infty} \frac{(1 / 2)_{i}(m / 2)_{i}}{(i !)^{2}} \\
& \times F\left(\frac{1}{2}+i, n ; 1+i ; \frac{4 a \varepsilon}{(a+\varepsilon)^{2}+C_{j}^{2}-b^{2}}\right)\left[\frac{4 a \varepsilon}{(a+\varepsilon)^{2}-b^{2}}\right]^{i} \\
V_{n}^{(j)}= & \pi \sum_{k=0}^{\infty} \sum_{i=0}^{\infty} \frac{C_{j}^{2 k+1}(2 k) !}{2^{2 k-1}(k !)^{2}(2 k+1)\left[(a+\varepsilon)^{2}-b^{2}\right]^{\frac{n}{2}}\left[(a+\varepsilon)^{2}+C_{j}^{2}-b^{2}\right]^{k+\frac{1}{2}}} \\
& \times \frac{(1 / 2)_{i}(n / 2)_{i}}{(i !)^{2}} F\left(\frac{1}{2}+i, k+\frac{1}{2} ; 1+i ; \frac{4 a \varepsilon}{(a+\varepsilon)^{2}+C_{j}^{2}-b^{2}}\right) \\
& \times\left[\frac{4 a \varepsilon}{(a+\varepsilon)^{2}-b^{2}}\right]^{i} .
\end{aligned}
$$

Finally, we substitute $U_{(m, n)}^{(j)}$ and $V_{n}^{(j)}$ for specific values of $j, m$ and $n$ into (10) to obtain the formulae for $T_{n}$, which are used to determine the interaction energy between an offset sphere and a finite length tube. The formulae for $T_{n}$ when $n=3,4,6,7,8,9$ and 10 are

$$
\begin{aligned}
& T_{3}=\frac{3}{8} V_{5}^{(2)}+\frac{1}{4} C_{2} U_{(2,2)}^{(2)}+\frac{3}{8} C_{2} U_{(4,1)}^{(2)} \\
& -\frac{3}{8} V_{5}^{(1)}-\frac{1}{4} C_{1} U_{(2,2)}^{(1)}-\frac{3}{8} C_{1} U_{(4,1)}^{(1)}, \\
& T_{4}=\frac{5}{16} V_{7}^{(2)}+\frac{1}{6} C_{2} U_{(2,3)}^{(2)}+\frac{5}{24} C_{2} U_{(4,2)}^{(2)}+\frac{5}{16} C_{2} U_{(6,1)}^{(2)} \\
& -\frac{5}{16} V_{7}^{(1)}-\frac{1}{6} C_{1} U_{(2,3)}^{(1)}-\frac{5}{24} C_{1} U_{(4,2)}^{(1)}-\frac{5}{16} C_{1} U_{(6,1)}^{(1)} \text {, } \\
& T_{6}=\frac{63}{256} V_{11}^{(2)}+\frac{1}{10} C_{2} U_{(2,5)}^{(2)}+\frac{9}{80} C_{2} U_{(4,4)}^{(2)}+\frac{21}{160} C_{2} U_{(6,3)}^{(2)} \\
& +\frac{21}{128} C_{2} U_{(8,2)}^{(2)}+\frac{63}{256} C_{2} U_{(10,1)}^{(2)}-\frac{63}{256} V_{11}^{(1)}-\frac{1}{10} C_{1} U_{(2,5)}^{(1)} \\
& -\frac{9}{80} C_{1} U_{(4,4)}^{(1)}-\frac{21}{160} C_{1} U_{(6,3)}^{(1)}-\frac{21}{128} C_{1} U_{(8,2)}^{(1)}-\frac{63}{256} C_{1} U_{(10,1)}^{(1)} \text {, }
\end{aligned}
$$




$$
\begin{aligned}
& T_{7}=\frac{231}{1024} V_{13}^{(2)}+\frac{1}{12} C_{2} U_{(2,6)}^{(2)}+\frac{11}{120} C_{2} U_{(4,5)}^{(2)}+\frac{33}{320} C_{2} U_{(6,4)}^{(2)} \\
& +\frac{77}{640} C_{2} U_{(8,3)}^{(2)}+\frac{77}{512} C_{2} U_{(10,2)}^{(2)}+\frac{231}{1024} C_{2} U_{(12,1)}^{(2)} \\
& -\frac{231}{1024} V_{13}^{(1)}-\frac{1}{12} C_{1} U_{(2,6)}^{(1)}-\frac{11}{120} C_{1} U_{(4,5)}^{(1)} \\
& -\frac{33}{320} C_{1} U_{(6,4)}^{(1)}-\frac{77}{640} C_{1} U_{(8,3)}^{(1)}-\frac{77}{512} C_{1} U_{(10,2)}^{(1)} \\
& -\frac{231}{1024} C_{1} U_{(12,1)}^{(1)} \text {, } \\
& T_{8}=\frac{429}{2048} V_{15}^{(2)}+\frac{429}{2048} C_{2} U_{(14,1)}^{(2)}+\frac{143}{1024} C_{2} U_{(12,2)}^{(2)} \\
& +\frac{143}{1280} C_{2} U_{(10,3)}^{(2)}+\frac{429}{4480} C_{2} U_{(8,4)}^{(2)} \\
& +\frac{143}{1680} C_{2} U_{(6,5)}^{(2)}+\frac{13}{168} C_{2} U_{(4,6)}^{(2)}+\frac{1}{14} C_{2} U_{(2,7)}^{(2)} \\
& -\frac{429}{2048} V_{15}^{(1)}-\frac{429}{2048} C_{1} U_{(14,1)}^{(1)}-\frac{143}{1024} C_{1} U_{(12,2)}^{(1)} \\
& -\frac{143}{1280} C_{1} U_{(10,3)}^{(1)}-\frac{429}{4480} C_{1} U_{(8,4)}^{(1)}-\frac{143}{1680} C_{1} U_{(6,5)}^{(1)} \\
& -\frac{13}{168} C_{1} U_{(4,6)}^{(1)}-\frac{1}{14} C_{1} U_{(2,7)}^{(1)} \text {, } \\
& T_{9}=\frac{6435}{32768} V_{17}^{(2)}+\frac{6435}{32768} C_{2} U_{(16,1)}^{(2)}+\frac{2145}{16384} C_{2} U_{(14,2)}^{(2)} \\
& +\frac{429}{4096} C_{2} U_{(12,3)}^{(2)}+\frac{1287}{14336} C_{2} U_{(10,4)}^{(2)}+\frac{143}{1792} C_{2} U_{(8,5)}^{(2)} \\
& +\frac{65}{896} C_{2} U_{(6,6)}^{(2)}+\frac{15}{224} C_{2} U_{(4,7)}^{(2)}+\frac{1}{16} C_{2} U_{(2,8)}^{(2)} \\
& -\frac{6435}{32768} V_{17}^{(1)}-\frac{6435}{32768} C_{1} U_{(16,1)}^{(1)}-\frac{2145}{16384} C_{1} U_{(14,2)}^{(1)} \\
& -\frac{429}{4096} C_{1} U_{(12,3)}^{(1)}-\frac{1287}{14336} C_{1} U_{(10,4)}^{(1)}-\frac{143}{1792} C_{1} U_{(8,5)}^{(1)} \\
& -\frac{65}{896} C_{1} U_{(6,6)}^{(1)}-\frac{15}{224} C_{1} U_{(4,7)}^{(1)}-\frac{1}{16} C_{1} U_{(2,8)}^{(1)} \text {, } \\
& T_{10}=\frac{12155}{65536} V_{19}^{(2)}+\frac{12155}{65536} C_{2} U_{(18,1)}^{(2)}+\frac{12155}{98304} C_{2} U_{(16,2)}^{(2)} \\
& +\frac{2431}{24576} C_{2} U_{(14,3)}^{(2)}+\frac{2431}{28672} C_{2} U_{(12,4)}^{(2)}+\frac{2431}{32256} C_{2} U_{(10,5)}^{(2)} \\
& +\frac{1105}{16128} C_{2} U_{(8,6)}^{(2)}+\frac{85}{1344} C_{2} U_{(6,7)}^{(2)}+\frac{17}{288} C_{2} U_{(4,8)}^{(2)} \\
& +\frac{1}{18} C_{2} U_{(2,9)}^{(2)}-\frac{12155}{65536} V_{19}^{(1)}-\frac{12155}{65536} C_{1} U_{(18,1)}^{(1)} \\
& -\frac{12155}{98304} C_{1} U_{(16,2)}^{(1)}-\frac{2431}{24576} C_{1} U_{(14,3)}^{(1)}-\frac{2431}{28672} C_{1} U_{(12,4)}^{(1)} \\
& -\frac{2431}{32256} C_{1} U_{(10,5)}^{(1)}-\frac{1105}{16128} C_{1} U_{(8,6)}^{(1)}-\frac{85}{1344} C_{1} U_{(6,7)}^{(1)} \\
& -\frac{17}{288} C_{1} U_{(4,8)}^{(1)}-\frac{1}{18} C_{1} U_{(2,9)}^{(1)} \text {. }
\end{aligned}
$$

We note that the analytical expressions for $U_{(m, n)}^{(j)}$ and $V_{n}^{(j)}$ are given by (22) and (23), respectively, and $C_{1}=-Z$ and $C_{2}=L-Z$.

\section{References}

Bailey WN (1972) Generalized hypergeometric series. Hafner Publishing Company, New York

Baowan D, Cox BJ, Hill JM (2012) Instability of $\mathrm{C}_{60}$ fullerene interacting with lipid bilayer. J Mol Model 18:549-557

Baowan D, Hill JM (2007) Force distribution for double-walled carbon nanotubes and gigahertz oscillators. Zeitschrift für Angewandte Mathematik und Physik 58:857-875

Baowan D, Peuschel H, Kraegeloh A, Helms V (2013) Energetics of liposomes encapsulating in silica nanoparticles. J Mol Model 19:2459-2472

Baowan D, Thamwattana N (2014) Modelling encapsulation of gold and silver nanoparticles inside lipid nanotubes. Phys A Stat Mech Appl 396:149-154

Baowan D, Thamwattana N, Hill JM (2007) Encapsulation of $\mathrm{C}_{60}$ fullerenes into single-walled carbon nanotubes: fundamental mechanical principles and conventional applied mathematical modeling. Phys Rev B 76:155411

Baowan D, Thamwattana N, Hill JM (2008) Suction energy and offset configuration for double-walled carbon nanotubes. Commun Nonlinear Sci Numer Simul 13:1431-1447

Cheng J, Zhu J, Liu B, Liao Z, Lai Z (2009) Structure of a selfassembled single nanotube of cyclo[(-d-Ala-1-Ala $\left.)_{4}-\right]$. Mol Simul 35(8):625-630

Cox BJ, Thamwattana N, Hill JM (2007a) Mechanics of atoms and fullerenes in single-walled carbon nanotubes. I. Acceptance and suction energies. Proc R Soc A Math Phys Eng Sci 463:461-476

Cox BJ, Thamwattana N, Hill JM (2007b) Mechanics of atoms and fullerenes in single-walled carbon nanotubes. II. Oscillatory behaviour. Proc R Soc A Math Phys Eng Sci 463:477-494

Cox BJ, Thamwattana N, Hill JM (2008) Orientation of spheroidal fullerenes inside carbon nanotubes with potential applications as memory devices in nano-computing. J Phys A Math Theor 41:235209

Delemotte L, Dehez F, Treptow W, Tarek M (2008) Modeling membranes under a transmembrane potential. J Phys Chem B 112:5547-5550

Dinca V, Kasotakis E, Catherine J, Mourka A, Ranella A, Ovsianikov A, Chichkov BN, Farsari M, Mitraki A, Fotakis C (2008) Directed three-dimensional patterning of self-assembled peptide fibrils. Nano Lett 8(2):538-543

Engels M, Bashford D, Ghadiri MR (1995) Structure and dynamics of self-assembling peptide nanotubes and the channel-mediated water organization and self-diffusion. A molecular dynamics study. J Am Chem Soc 117(36):9151-9158

Ghadiri MR, Granja JR, Milligan RA, McRee DE, Khazanovich N (1993) Self-assembling organic nanotubes based on a cyclic peptide architecture. Nature 366:324-327

Girifalco LA (1991) Interaction potential for carbon $\mathrm{C}_{60}$ molecules. J Phys Chem 95:5370-5371

Gradshteyn IS, Ryzhik IM (2000) Table of integrals, series, and products, 6th edn. Academic Press, San Diego

Hwang H, Schatz GC, Ratner MA (2009) Coarse-grained molecular dynamics study of cyclic peptide nanotube insertion into a lipid bilayer. J Phys Chem A 113:4780-4787

Khalfa A, Treptow W, Maigret B, Tarek M (2009) Self assembly of peptides near or within membranes using coarse grained MD simulations. Chem Phys 358:161-170

Khurana E, DeVane RH, Kohlmeyer A, Klein ML (2008) Probing peptide nanotube self-assembly at a liquid-liquid interface with coarse-grained molecular dynamics. Nano Lett 8:3626-3630

Kim HS, Hartgerink JD, Ghadiri MR (1998) Oriented self-assembly of cyclic peptide nanotubes in lipid membranes. J Am Chem Soc 120:4417-4424 
Liu J, Fan J, Tang M, Zhou W (2010) Molecular dynamics simulation for the structure of the water chain in a transmembrane peptide nanotube. J Phys Chem A 114:2376-2383

Raghavender US, Aravinda KS, Shamala N, Balaram P (2010) Hydrophobic peptide channels and encapsulated water wires. J Am Chem Soc 132:1075-1086

Raghavender US, Aravinda S, Shamala N, Kantharaju, Rai R, Balaram P (2009) Characterization of water wires inside hydrophobic tubular peptide structures. J Am Chem Soc 131:15130-15132

Rahmat F, Thamwattana N, Cox BJ (2011) Modelling peptide nanotubes for artificial ion channels. Nanotechnology 22:445707 (8 pp.)

Rappe AK, Casewit CJ, Colwell KS, Goddard WA III, Skiff WM (1992) UFF, a full periodic table force field for molecular mechanics and molecular dynamics simulations. J Am Chem Soc 114:10024-10035

Scanlon S, Aggeli A (2008) Self-assembling peptide nanotubes. Nano Today 3(3-4):22-30

Shoseyov O, Levy I (eds) (2008) NanoBiotechnology bioinspired devices and materials of the future. Humana Press, Totowa

Song X, Fan J, Liu D, Li H, Li R (2013) Molecular dynamics study of $\mathrm{Na}^{+}$transportation in a cyclic peptide nanotube and its influences on water behaviors in the tube. J Mol Model 19(10):4271-4282

Tarek M, Maigret B, Chipot C (2003) Molecular dynamics investigation of an oriented cyclic peptide nanotube in DMPC bilayers. Biophys J 85:2287-2298

Thamwattana N, Baowan D, Hill JM (2009) Continuum modelling for interactions between fullerenes and other carbon nanostructures. J Comput Theor Nanosci 6:972-984

Tiangtrong P, Baowan D (2014) Encapsulation behaviours of nanoparticles entering two-section carbon nanotubes. J Math Chem 52:489-503

Zhang S (2003) Building from the bottom up. Mater Today 6(5):20-27

Zhao X, Zhang S (2007) Designer self-assembling peptide materials. Macromol Biosci 7(1):13-22

Zhu J, Cheng J, Liao Z, Lai Z, Liu B (2008) Investigation of structures and properties of cyclic peptide nanotubes by experiment and molecular dynamics. J Computer-Aided Mol Design 22:773-781 